\title{
The Role of circulating sTWEAK in the pathogenesis of Hashimoto's Thyroiditis and its relationship with other cytokines: A pilot study
}

Mustafa Altay ${ }^{1}$, İhsan Ateş ${ }^{2}$, Fatma Meriç Yılmaz ${ }^{3}$, Canan Topçuoğlư ${ }^{3}$, Mustafa Kaplann ${ }^{4}$ Fatma Aybala Altay ${ }^{5}$

${ }^{1}$ Keçiören Education and Research Hospital, Department of Endocrinology and Metabolism, Ankara, Turkey

2Numune Education and Research Hospital, Department of Internal Medicine, Ankara, Turkey

${ }^{3}$ Numune Education and Research Hospital, Department of Biochemistry, Ankara, Turkey

4Yüksek intisas Education and Resarch Hospital, Department of Gastroenterology, Ankara, Turkey

${ }^{5}$ Dışkapı Education and Research Hospital, Department of Infectious Disease and Clinical Microbiology, Ankara,

Turkey

\section{OBJECTIVE and METHODS}

We aimed to investigate the role of STWEAK in the pathogenesis of Hashimoto's Thyroiditis(HT) and its relationship with IL-12, IL 17-A and TGF- $\beta 1$.

Serum TSH, TT4, anti-TG and anti-TPO were measured and thyroid USG was performed to both group. In addition serum samples were collected and STWEAK, IL 17-A, IL-12 and TGF$\beta 1$ were measured.

\section{PATIENTS}

Sixty patients(20 patients were euthyroid, 20 patients were subclinical hypothyroidism and other 20 were overt hypothyroidism), who were newly diagnosed with $\mathrm{HT}$ and did not receive any treatment, and 20 patients with no known disease as the healthy control group were included in the study.

\section{RESULTS}

The HT group had lower levels of sTWEAK and TGF- $\beta 1$, but had higher levels of IL-12 and IL 17-A as compared to the control group. Of these, only the difference between IL 17-A levels reached the statistical significance(2.1(1.1-10.7) vs $1.8(1.2-2.3)) p<0,001)$. The overt hypothyroidism group had significantly higher levels of IL-12 than those of other groups. All of the subgroups had significantly higher levels of IL 17-A than those of control group. sTWEAK was negatively correlated with IL 17-A in the overt hypothyroidism.

\section{DISCUSSION}

We achieved data that support the role of IL-12 and IL17-A in the pathogenesis of HT but did not find significant differences between the sTWEAK level and the groups. Also, no significant relationship was identified between the sTWEAK level and either the thyroid autoantibodies or the cytokine levels of other pathways. Further studies are needed to present the effect of sTWEAK level on the autoimmune diseases.
Table 1. Comparison of subgroups of the HT patients and controls

\begin{tabular}{|c|c|c|c|c|c|}
\hline & Control & Euthyroid & S. Hypothyroid & O. Hypothyroid & p value \\
\hline Age & $\begin{array}{|ll|}35.3 & 7.5 \\
\end{array}$ & \begin{tabular}{|ll}
32.5 & 8.2
\end{tabular} & 30.99 .9 & \begin{tabular}{|ll}
31.9 & 8.2 \\
\end{tabular} & 0.9 \\
\hline $\operatorname{Sex}(\mathbf{M} / \mathbf{F})$ & $2 / 18$ & 1/19 & 2/18 & $3 / 17$ & \\
\hline BMI & 24.62 .3 & 23.62 & 25.13 & $\begin{array}{ll}24 & 3.3\end{array}$ & 0.6 \\
\hline Systolic BP(mmHg) & $110(90-125)$ & $118.5(100-128)$ & $110(90-132)$ & $116(90-135)$ & 0.08 \\
\hline $\begin{array}{l}\text { Diastolic } \\
\text { BP(mmHg) }\end{array}$ & $70(60-80)$ & $62.5(56-87)$ & $70(53-90)$ & $72.5(60-85)$ & 0.02 ce,f \\
\hline TSH $(\mu \mathrm{IU} / \mathbf{m L})$ & $1.6(0.9-3.5)$ & $2.1(0.9-4.2)$ & 7.1(4.6-14.5) & 33.3(9.6-207.6) & $, \mathrm{b}, \mathrm{c}, \mathrm{d}, \mathrm{d}, \mathrm{f}$ \\
\hline $\mathrm{fT} 3(\mathrm{pg} / \mathrm{mL})$ & $\begin{array}{ll}2.8 & 0.5\end{array}$ & - & $\begin{array}{ll}3 & 0.4\end{array}$ & 2.20 .8 & $<0.01$ \\
\hline fT4(ng/dL) & 1.20 .5 & $\begin{array}{ll}1.2 & 0.1 \\
\end{array}$ & 1.10 .1 & $\begin{array}{ll}0.6 & 0.1\end{array}$ & $<0.01^{b}$ \\
\hline Anti-TG(IU/mL) & $7.6(2.4-26)$ & $207.1(16.7-1538)$ & $219(10-3910)$ & 341(59.4-7411) & $<, \mathbf{b}, \mathbf{c}$ \\
\hline Anti-TPO (IU/mL) & 3.9(1-39) & 43.1(5-443) & $119(4-9578)$ & $515.5(6-7977)$ & $<, b, b, c, e, f$ \\
\hline TWEAK (pg/mL) & 838.5236 .5 & 771.4 215.4 & $\begin{array}{lll}888.2 & 374.4\end{array}$ & $687.6 \quad 153.3$ & $0.04^{\mathrm{d}}$ \\
\hline TGF-p1 (ng/mL) & $29.4(0.3-153.1)$ & $7.3(0.5-104.6)$ & $27(0.6-205.8)$ & $15.7(0.5-97.5)$ & 0.2 \\
\hline IL-12(pg/mL) & $2.7(1.9-4.2)$ & $2.7(2.2-5.8)$ & $3.1(2.1-29.1)$ & $3.6(2.4-7.6)$ & $<0.01$ b,f \\
\hline IL 17-A(pg/mL) & $1.8(1.2-2.3)$ & $2.3(1.6-10.7)$ & 2(1.1-2.7) & $2.3(1.5-7.8)$ & $<, \mathbf{b}, \mathbf{c}, \mathbf{e}$ \\
\hline
\end{tabular}

a: Control vs $\mathrm{SH}, \mathrm{b}$ : Control vs $\mathrm{OH}, \mathrm{c}$ : Control vs Euthyroid HT, d:SH vs $\mathrm{OH}$, e:SH vs Euthyroid HT, f:OH vs Euthyroid HT

Abbreviations: $\mathrm{BMl}$ :body mass index, $\mathrm{BP}$ : blood pressure, $\mathrm{TSH}$ : thyroid stimulating hormone, anti-TG: anti-thyroglobuline, anti-TPO: anti-thyroidperoxidase, TGF- $\beta 1$ : transforming growth factor beta-1, $\mathrm{SH}$ : subclinical hypothyroidism, $\mathrm{OH}$ : overt hypothyroidism, $\mathrm{HT}$ : Hashimoto's thyroiditis 